\title{
Modeling Degradation of Metallic Surfaces By First Principles Calculations: A Case Study for Al, Au, Ag, and Pd
}

\author{
Teresita Carrasco ${ }^{1}$, Joaquín Peralta ${ }^{1}$ and Scott R. Broderick ${ }^{2}$ \\ 1. Departamento de Física, Facultad de Ciencias Exactas, Universidad Andrés Bello, Santiago, Chile. \\ 2. Dept. of Materials Designs and Innovation-University at Buffalo: State University of New York, USA.
}

Under the effects of an extreme electric field, the atoms on a metallic surface evaporate by breaking their bonds with the surface. In this work, we present the effects of a high electric field, by mean of computational simulations, for different metallic surface chemistries: $\mathrm{Al}, \mathrm{Au}, \mathrm{Ag}$ and $\mathrm{Pd}$. The presence of high electric fields on the order of hundred $\mathrm{MeV} / \mathrm{m}$ is becoming increasingly common as science and engineering continues to push characterization boundaries. These kinds of electric fields are observed in particle accelerators, field emission microscopy, field ion microscopy, and atom probes, among others. To model this bond breaking procedure (ie. field evaporation), we use density functional theory (DFT) performed through the Quantum-Espresso [1] simulation (QE) package, which incorporates the electric field by adding a saw-like function into the Hamiltonian [2]. This approach, known as dipole correction, was applied to all simulations as is implemented in the QE package.

In this work, we calculate the evaporation field $\left(F_{e}\right)$, which corresponds to the mean field at which atoms can break their bonds from the surface and evaporate. This result is compared with experimental data from atom probe tomography (APT) and computational data from prior simulations [3-5]. In order to determine the $F_{e}$ value, two main surface orientations, $\langle 111\rangle$ and $\langle 100\rangle$, have been considered. Adatoms are displaced from these pristine surfaces under the action of different values of applied electrics fields, characterizing the system energetics. When the electric field increases, the hump height decreases. When the electric field is close to $F_{e}$, the hump disappears (corresponding to the activation energy) and the ad-atom is free from the surface. The $F_{e}$ value is essential in (i) understanding surface bonding as a function of electric field, (ii) improving the reconstruction of collected APT data, and (iii) modeling the degradation of materials under extreme conditions.

The structures were built using 25 and 21 atoms for the $\langle 111\rangle$ and $\langle 100\rangle$ surfaces, respectively. Figure 1a shows a representative structure for gold in the $\langle 111\rangle$ direction. All slabs were located in the middle of a supercell with a vacuum region of $15 \AA$ to the borders of the supercell in the z-axis. The calculations were performed with QE under generalized gradient approximation (GGA). The energy cut-off used for each atomic species were 25, 40, 25, and $35 \mathrm{Rydberg}$ for $\mathrm{Ag}, \mathrm{Al}, \mathrm{Au}$, and $\mathrm{Pd}$, respectively. The k-point number is $12 \times 12 \times 12$ for Au case, and $8 \times 8 \times 8$ for all of the rest. To determine the field evaporation value for each surface, different electric field were applied ranging from 0 to $54 \mathrm{~V} / \mathrm{nm}$. For each electric field applied, a single ad-atom on the surface was displaced at twelve different distances, and then the energy of the system was calculated. The obtained energies at different electric fields and distances shows the variation of the energy curve. Results for the case of $\mathrm{Al}$ are presented in Figure $1 \mathrm{~b}$. In all cases, as the electric fields are increased, the hump is reduced. The evaporation field is the electric field value at which the hump practically disappears [5], and is different for each type of surface. In order to determine the hump for each energy versus ad-atom position curve, we fit the results numerically with a standard seven parameter equation [5] and then the hump variation is determined by fitting a generalized logistic function (or Richard's curve) [5]. Figure 1c shows the variation of the hump as the electric field increases. 
The results for the different surfaces (Table 1) shows that the scheme used gives us a good comparison with both experimental and other computational values. We can observe that some $F_{e}$ values are overestimated in our results, which may be partially due to overestimation of the energy by the generalized gradient approximation (GGA) technique, but mainly result from the use of a pristine surface, which is not the case for samples used in experiments. These results provide us valuable information about the response of these materials under the effects of extremely high electric field, and therefore the design of materials for used in extreme condition environments[6].

\section{References:}

[1] P. Gianozzi, S. Baroni, N. Bonini, et al, Journal of Physics: Condensed Matter 29 (1009) p. 395502.

[2] L. Bengsston, Physical Review B 59 (1999) p. 12301.

[3] H.J. Kreuzer, Physical Review B 45 (1992) p.12050.

[4] C.G. Sanchez, A.Y. Lozovoi, A. Alavi, Molecular Physics 102 (2004) p. 1045.

[5] J. Peralta, S.R. Broderick, K. Rajan. Ultramicroscopy 132 (2013) p 143.

[6] TC \& JP acknowledges funding from Proyecto Fondecyt Iniciacion 11130501.

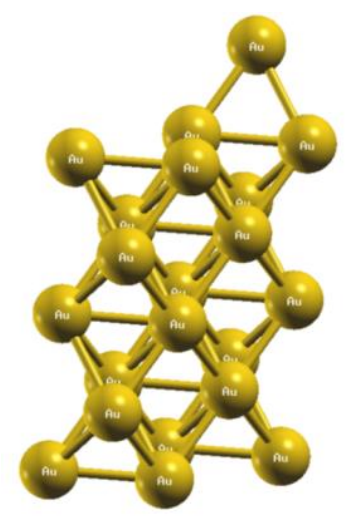

(a)

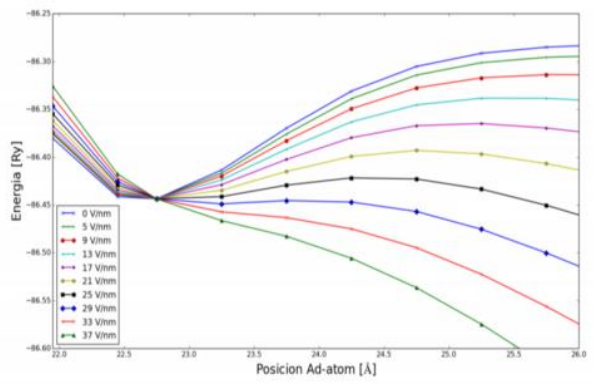

(b)

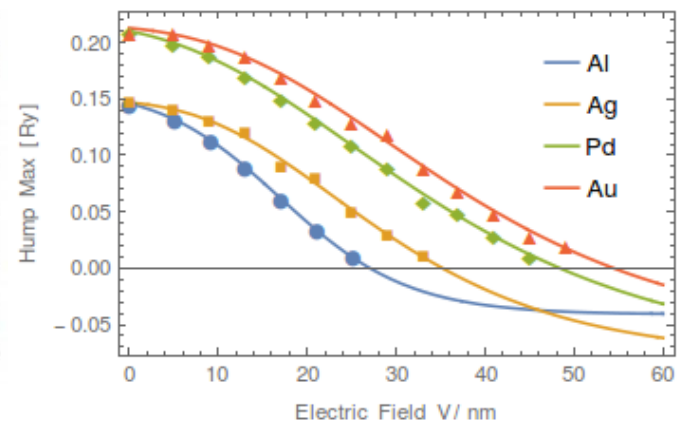

(c)

Figure 1. (a) A representative $<100>-A u$ structure with an ad-atom on the surface in the z-direction. (b) Variation of the energy as a function of electric field and distance. (cc) Variation of the hump of the energy-curve as a function of electric field.

\begin{tabular}{llll}
\hline Metal & Fe $<\mathbf{1 1 1}>[\mathbf{V} / \mathbf{n m}]$ & $\mathbf{F e}<\mathbf{1 0 0}>[\mathbf{V} / \mathbf{n m}]$ & Experimental $[\mathbf{V} / \mathbf{n m}]$ \\
\hline Ag & 35.52 & 33.30 & 24 \\
Al & 27 & 27 & 19 \\
Au & 54.4 & 52.4 & 53 \\
Pd & 48.4 & 50.9 & 37 \\
\hline
\end{tabular}

Table 1. The field evaporation values determined by our technique for different metallic surfaces. 